\title{
Caracterización de las arcillas utilizadas en alfarería en tres corregimientos del municipio de Valledupar Cesar, Colombia
}

\section{Artículo de investigación}

\section{María Cristina Cotes de Álvarez}

Universidad Popular del Cesar, Colombia

mariacotes@unicesar.edu.co

Recibido: 10 de junio de 2017

Aprobado: 20 de septiembre de 2017

Cómo citar este artículo: Cotes de Álvarez, María Cristina. (2018) Caracterización de las arcillas utilizadas en alfarería en tres corregimientos del municipio de Valledupar - Cesar, Colombia. Calle14: revista de investigación en el campo del arte, 13 (23) pp. 198-209 DOI: pendiente 


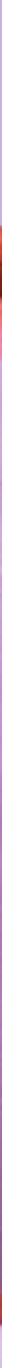


Caracterización de las arcillas utilizadas en alfarería en tres corregimientos del municipio de

Valledupar - Cesar, Colombia

\begin{abstract}
Resumen
La evidencia de una tradición en vía de extinción y la necesidad de tecnologías adecuadas conduce a la búsqueda de mecanismos que permitan optimizar la calidad del trabajo alfarero en tres corregimientos de Valledupar, costa norte de Colombia. Para ello, se analizaron propiedades físicas, térmicas y trabajabilidad de la arcilla y así reconocer cualidades y características de seis muestras en un total de diez pruebas. A través de metodologías con carácter experimental y multidisciplinario se abrieron diálogos entre saberes mediante talleres de diagnóstico con niños, jóvenes y adultos, quedando expuestas fallas de producción y cocción. Con el fin de suplir estas falencias, se construyó de manera experimental un horno botella de tiro directo a leña en uno de los corregimientos. Es necesario, aún, atinar el nivel calórico adecuado en este horno y ajustar el manejo de temperaturas. Además, se aporta un sumario formativo y orientador que consigna la aplicación científica del proyecto.
\end{abstract}

\title{
Palabras clave
}

Arcilla, caracterización, cocción, rescate, tradición.

Characterization of the clays used in pottery in three settlements in the municipality of Valledupar Cesar, Colombia

\begin{abstract}
The evidence of an endangered tradition and the need for adequate technologies leads to the search for mechanisms that allow optimizing the quality of pottery work in three settlements in Valledupar, Colombia's northern coast. For this, physical, thermal and workability properties of the clay were analyzed in order to recognize the characteristics and qualities of six samples in a total of ten tests. Experimental and multidisciplinary methodologies opened dialogues between forms of knowledge, through diagnostic workshops with children, the youth and adults, exposing errors in production and cooking. In order to compensate for these shortcomings, a bottletype furnace for wood was built in an experimental manner in one of the settlements. It is also necessary to check the appropriate calorie level in this oven and adjust the management of temperature. In addition, a formative and guiding summary is provided that indicates the scientific applications of the project.
\end{abstract}

\section{Keywords}

Clay, characterization, cooking, rescue, tradition.

Caractérisation des argiles utilisées en poterie dans trois établissements de la municipalité de Valledupar - Cesar, Colombie

\section{Résumé}

La preuve d'une tradition menacée et le besoin de technologies adéquates conduisent à la recherche de mécanismes permettant d'optimiser la qualité de la poterie dans trois villages de Valledupar, sur la côte nord de la Colombie. Pour cela, les propriétés physiques, thermiques et de maniabilité de l'argile ont été analysées afin de reconnaître les caractéristiques et les qualités de six échantillons dans un total de dix essais. Des méthodologies expérimentales et pluridisciplinaires ont ouvert le dialogue entre les formes de connaissance, à travers des ateliers de diagnostic avec les enfants, les jeunes et les adultes, exposant les erreurs dans la production et la cuisson. Afin de pallier ces insuffisances, un four à bouteilles de bois a été construit de manière expérimentale dans l'un des établissements. Il est également nécessaire de vérifier le niveau 
de calorie approprié dans ce four et d'ajuster la gestion de la température. En outre, un résumé formatif et directeur est fourni qui indique les applications scientifiques du projet.

\section{Mots clés}

Argile, caractérisation, cuisine, sauvetage, tradition.

Caracterização das argolas usadas em cerâmica em três assentamentos no município de Valledupar Cesar, Colômbia

\section{Resumo}

A evidência de uma tradição em extinção e a necessidade de tecnologias adequadas levam à busca de mecanismos que permitam otimizar a qualidade do trabalho de cerâmica em três assentamentos em Valledupar, na costa norte da Colômbia. Para isso, as propriedades físicas, térmicas e de trabalho da argila foram analisadas para reconhecer as características e qualidades de seis amostras em um total de dez testes. As metodologias experimentais e multidisciplinares abriram diálogos entre formas de conhecimento, através de oficinas de diagnóstico com crianças, jovens e adultos, expondo erros na produção e no cozido. Para compensar essas falhas, um forno tipo garrafa para madeira foi construído de forma experimental em um dos assentamentos. Também é necessário verificar o nível de calorias apropriado neste forno e ajustar o gerenciamento da temperatura. Além disso, é fornecido um resumo formativo e orientador que indica as aplicações científicas do projeto.

\section{Palavras-chave}

Argila, caracterização, culinária, resgate, tradição.

\section{Imasami rurarkakuna turu alpa tsabaju kai kimsa suti alpa atun Valledurpamanda- Cesar, Colombia}

\section{Maillallachiska:}

Kauachirimi kai puchukaikuna i imami ministiri kai tsabajukunawa aidami i maitsu alpa turua tsabajadurkunapa kai alpa Valleduparmanda suti Colombiamanda a kai alpa Valleduparmanda suti Colombiamanda kagkuna. Iurrarispa churarikami imasa kagta, chiri u kunu kagta i kawachispa kai alpa turua maitukumi suma kai sugta Tukui chunga ruraska i kawachiska. Kai iachachikunapi suma kagpi kaiarimi parlukuna sug ruraikuna i imasami kariwambra, ruraskaiatata i musu atunkuna rurarkuna kai tuto alpa ianespa puchukarkuna. Kai mana allilla ruraspa, ruraresk sug tulpa kasudiru chi ukuma luarmi. Kai chasa rurai ministimi sug atun maitsu chasami suma rurankuna kai iuiaipi.

\section{Rimangapa Ministidukuna:}

Prácticas artísticas, kawachirii, ianurii, tarinakuii, Nukanchipa kustumbri. 


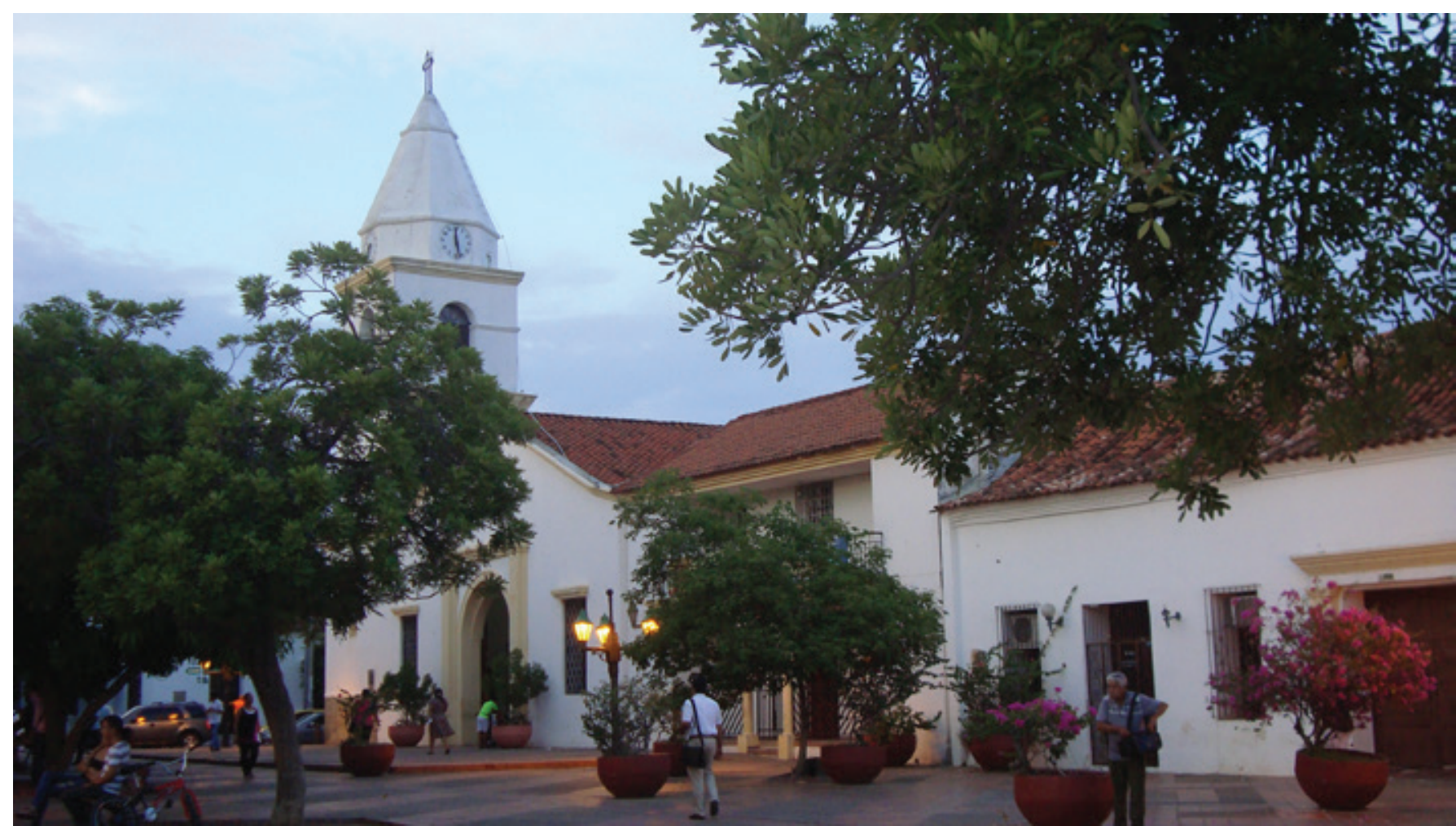

Plaza Alfonso López Valledupar. Fotografía: María Cristina Cotes

\section{Introducción}

Patillal y Guacoche son dos corregimientos del municipio de Valledupar, en la costa norte de Colombia, donde las mujeres trabajaban la alfarería, elaborando utensilios de uso doméstico, tales como ollas, tinajas, tiestos para tostar el café, asador de arepas... En Patillal, era el medio de supervivencia para algunas mujeres: los hombres aportaban a este oficio la preparación de la arcilla y la comercialización de la loza, sacándolas a lomo de burro por toda la provincia; pero las señoras que gozaban de recursos económicos, no lo hacían por negocio, solo para suplir la necesidad de su hogar, regalar y complacer a sus amistades.

Gozaban del privilegio de no tener afán en ejercer esta labor, por lo tanto, tenían la disponibilidad del tiempo para madurar y trabajar la arcilla, pausadamente. Esto beneficiaba la producción, por eso llegaron a hacer las mejores ollas de la región, bien estructuradas y de excelente calidad. La única decoración utilizada es el llamado barniz que extraían del Cerrito de las Cabras, en Patillal. Esta es una arcilla de tipología arenosa de color rojo a la cual adicionaban agua, la maduraban por unos días, luego bruñían y sellaban con una piedra lisa. La quema le proporcionaba un fino brillo natural y un color ocre muy bonito: "quedaban esos utensilios con un brillo... como si fueran esmaltados", relataron los ancianos cuando conversamos con ellos. Hoy día los resultados son bastante diferentes. Avances tecnológicos de la vida moderna han anulado la práctica artesanal. Esta ha dejado de ser competitiva en calidad y en producción, frente a otras que sí han recibido de instituciones gubernamentales el impulso necesario para salir adelante.

Por otra parte, el corregimiento de Guacoche del municipio de Valledupar, en la costa norte de Colombia, es un centro alfarero que otrora fue reconocido como uno de los mayores productores de tinajas del departamento. Las autoridades del pueblo organizan con apoyo institucional el Festival de la Tinaja, que se lleva a cabo a mediados del mes de diciembre, con el propósito de promover la enseñanza de técnicas ancestrales. En este desarrollan talleres y concursos que buscan estimular e impulsar la producción artesanal como un importante aporte para la conservación de la tradición. Hoy día, ya no se realizan los talleres en este festival. Según el criterio de algunas artesanas, el festival ha perdido el Norte. Afirman que no se les tiene en cuenta y el evento es solo de música vallenata; consideran que no hay estímulos para continuar y cultivar la labor, y por lo tanto han comenzado a buscar otras alternativas laborales. 


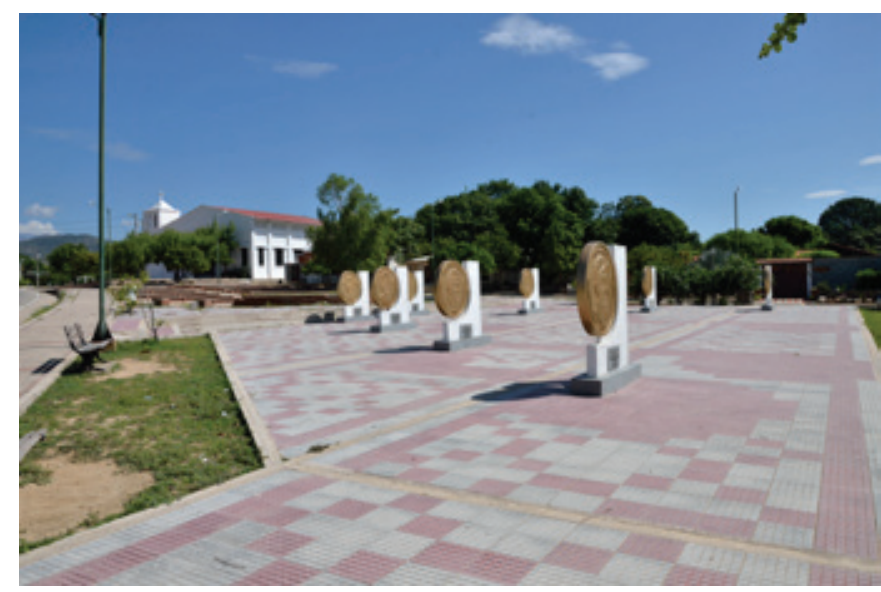

Plaza de las monedas. Patillal. Fotografía: María Cristina Cotes

En cuanto al corregimiento de Valencia de Jesús del municipio de Valledupar, en la costa norte de Colombia, este se ha caracterizado por su tradición alfarera. En él se fabrican ladrillos y tejas de barro, contribuyendo así al desarrollo de la infraestructura urbana en la región. Este es en un trabajo propiamente varonil, donde las mujeres no tienen oportunidades para desarrollarse laboralmente. Por ello. Artesanías de Colombia se interesó en impulsar el quehacer femenino para que se complementara con el masculino, y en el año de 1989 inició una serie de actividades con este fin. Entre ellas, una labor social estimulante que abarcaba a toda la familia, seguida de talleres mixtos de cerámica. Estos los desarrollé durante un año y medio. Allí se dejó trasmitida la inquietud: dos mujeres y un señor decidieron continuar con esta labor. Después de un trabajo continuo de muchos años, por diferentes motivos (como carencia de acompañamiento solidario, motivacional, social) renunciaron al oficio y hoy día ninguno de los tres se dedica a esta actividad.

Ser insensible ante esta situación no es la intención, por consiguiente se aportan técnicas y orientación desde la academia, intercambiando y difundiendo experiencias y saberes para contribuir a que el problema social sea menor.

\section{Una tradición en vía de extinción}

Tal vez la mayor debilidad en continuar con la tradición alfarera no radica en los factores técnico-productivos, sino en la ausencia de conocimiento de la mayoría de los miembros de la comunidad sobre el origen de su propio trabajo. Muchos integrantes de estas sociedades crecieron sin la posibilidad de conocer y mucho menos aprender sus tradiciones ancestrales. Dice Javier Marías

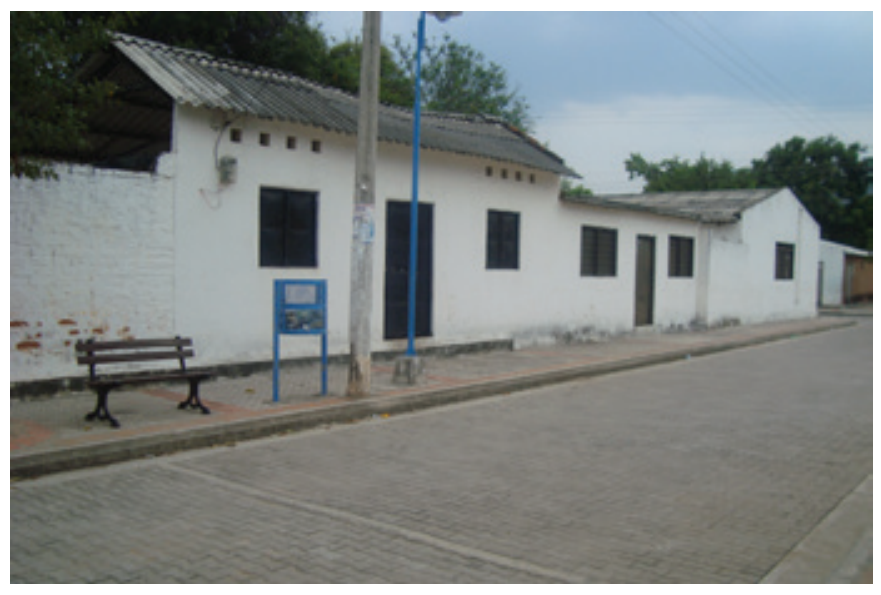

Casa en Guacoche. Fotografía: María Cristina Cotes

"Y olvidamos casi siempre que las vidas de las personas no son sólo eso: cada trayectoria se compone también de nuestras pérdidas y nuestros desperdicios, de nuestras omisiones y nuestros deseos incumplidos, de lo que una vez dejamos de lado o no elegimos o no alcanzamos, de las numerosas posibilidades que en su mayoría no llegaron a realizarse - todas menos una, a la postre-, de nuestras vacilaciones y nuestras ensoñaciones, de los proyectos frustrados y los anhelos falsos o tibios, de los miedos que nos paralizaron, de lo que abandonamos o nos abandonó a nosotros" (Parra A., 1998). Es este el origen decadente de esta tradición alfarera, porque va más allá de las faltas de metodologías, es probable que se trate de una causa social, cultural, humana, de estima, aspiraciones, de oportunidades, o incluso de apoyo institucional, o de direccionamiento cuando les brindan aportaciones, para rescatar la tradición.

Por ejemplo, la práctica artesanal en Guacoche ha despertado el interés del gobierno nacional y de organizaciones internacionales, quienes han contribuido con infraestructura, equipos y capacitación, fortaleciendo sus espacios socio-organizativos, con una sociedad de alfareros - sin indicarles cómo opera el sistema asociativo. Por otra parte, el enfoque aplicado no es el más indicado: les han implantado un sistema productivo que funciona muy bien en otras regiones del país, pero no se tuvo en cuenta la idiosincrasia, las necesidades, ni la identidad cultural regional. Por ello, desestiman los recursos otorgados y siguen limitados, elaborando escasos utensilios domésticos de poca durabilidad y terminados toscos, cuyos consumidores en mayor medida son los indígenas de la Sierra Nevada de Santa Marta y en menor proporción los del mercado público de Valledupar. 


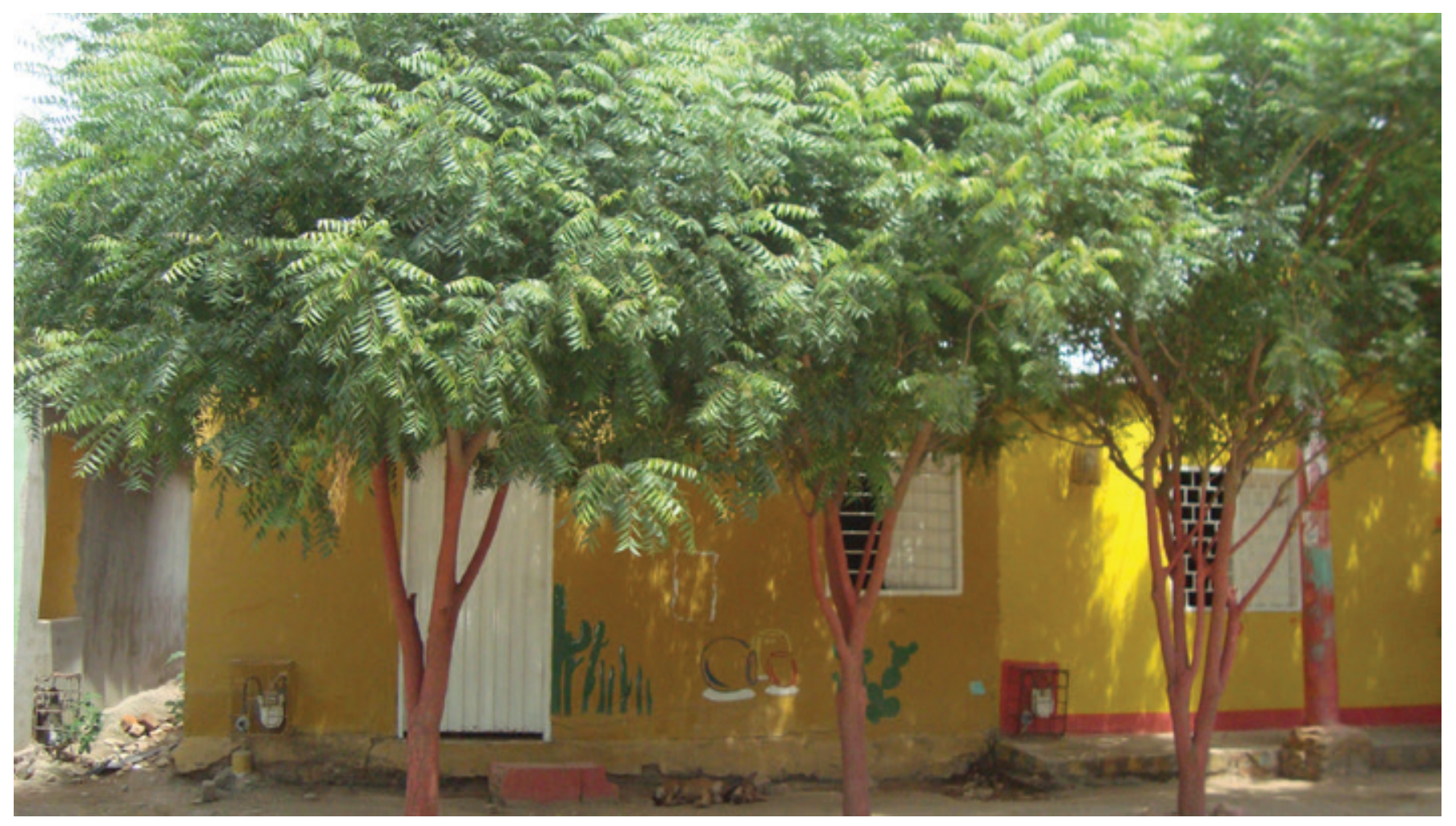

Calle en Valencia de Jesús. Fotografía: María Cristina Cotes

Pretendimos conocer el pensamiento y el querer de las comunidades, preguntándoles ¿qué quieren?, para encaminarlos, en él cómo lograrlo. Daniel Ariza expresa: "decidí crear inicialmente una acción dialogada entre diferentes autores y yo" (...) más adelante dice: "afiné mi idea sobre la configuración, no de un dialogo, sino de una conversación que no tuviera parlamentos definidos, sino que se fuera construyendo a través de la narración de pasajes de los ancianos" (Ariza, 2014). Hoy se les dice personas de la tercera edad —me gusta más, la palabra anciano: me transporta a la sabiduría, experiencia, saber y entender. Por consiguiente, se programaron talleres de diagnóstico para intimar con el estilo de trabajo de hombres y mujeres de cada corregimiento que dominan y practican el oficio: ellos enseñaron la metodología tradicional bajo mi tutoría y acompañamiento. Promovimos la participación de niños, jóvenes y adultos en grupos heterogéneos, donde existiera un intercambio y transferencias de saberes: anciana de 78 años enseña a su bisnieta de 4 . Allí se sembró una semilla que a su tiempo dará fruto para que la tradición no se esfume. Se identificaron procesos, técnicas y producción; y quedaron expuestas, fallas metodológicas.

Se detectó, además, que las personas interesadas en aprender este oficio ancestral deben atender prioritariamente sus necesidades básicas, por lo que toda actividad adicional representa un impedimento para suplirlas. Por lo tanto, consideramos necesaria la creación de nuevas fuentes de trabajo. Hacer ollas o tinajas de barro, en estas apartadas poblaciones, en momentos de avances científicos, tecnológicos e industriales, con presencia multicultural, desde todas las esferas del mundo —la globalización, el libre comercio-, convierten esta tarea de recuperación de la práctica artesanal, en un reto. Cuando Cleverth Cárdenas (2015) se refiere a los aymaras urbanos, dice que son migrantes del campo que han desarrollado una cultura muy compleja: porque en algunos casos niegan su procedencia, por la fuerte carga ideológica de la modernidad. Según él, esto al mismo tiempo los afirma de modo indefinido como que otros valores y principios apuntan a la reafirmación de su identidad nacional. Le parece interesante ver la articulación estratégica, la lógica para concluir y afirmarse como sujetos nacionales, folcloristas y migrantes. Este concepto identifica el fenómeno presentado en nuestra provincia. Por tal motivo, perseveramos por esta vitalidad artesanal que está a punto de extinguirse y que ahora es improbable que resurja debido a la falta de voluntad política y a las condiciones físicas, económicas y materiales. La determinación es acompañarles y aportar en la medida que nuestras posibilidades lo permitan, hasta que esto 


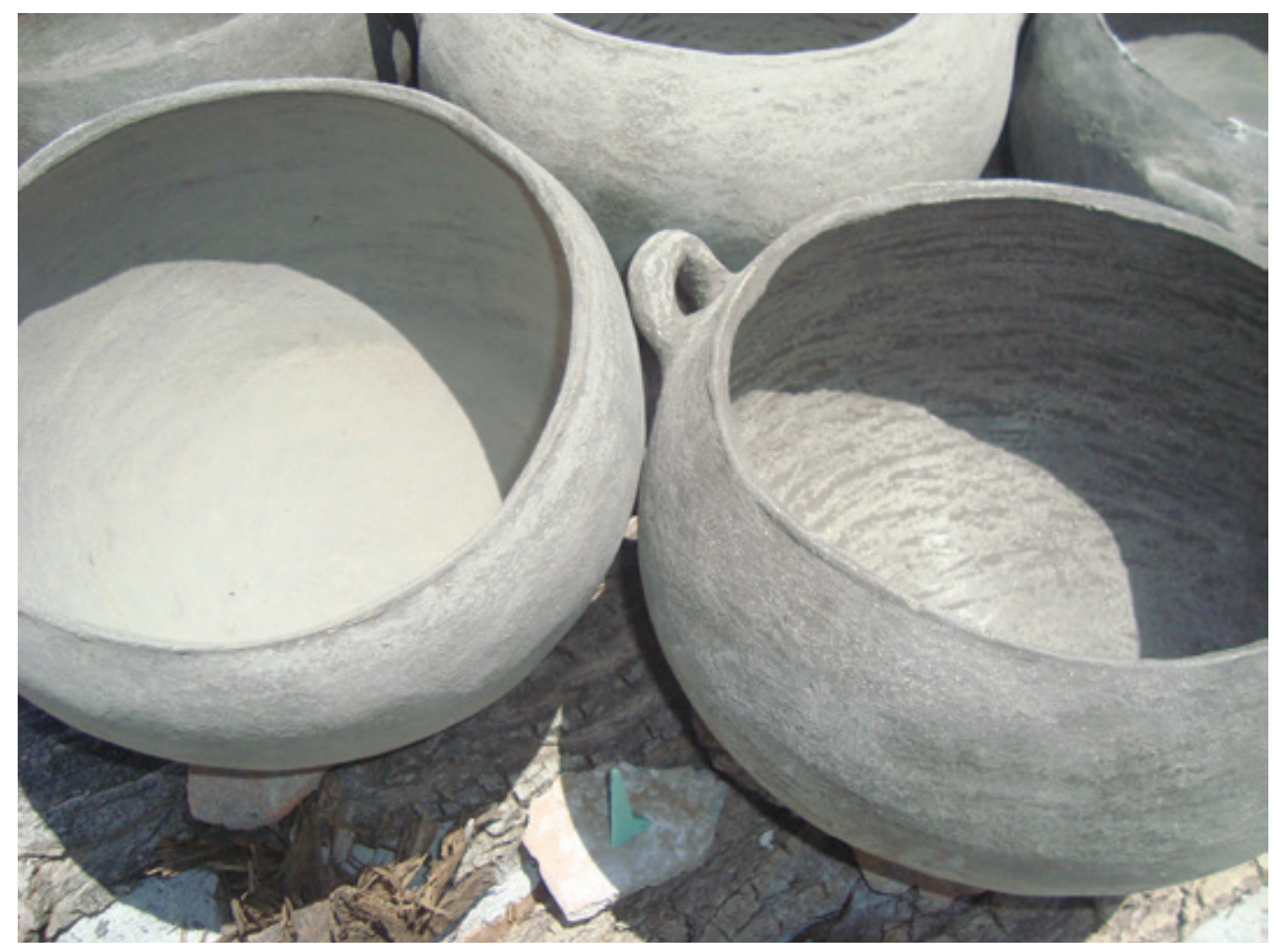

Ollas de barro listas para cocción. Fotografía: María Cristina Cotes

se logre. Procurando salvaguardar, estimular la creatividad y fomentar la innovación en la fabricación de los productos; valorando los vínculos de la alfarería con el universo presente, manteniendo sus costumbres, pero actualizando su adaptación y permanencia en la vida moderna, donde lo tradicional se posicione dentro de un estilo actual y de vanguardia.

\section{La academia tras la calidad y el rescate de una tradición}

¿Se podrá lograr un producto de buena calidad? ¿Es posible el rescate de esta tradición? La respuesta, primero, sin que ello signifique que sea la definitiva, es la aplicación de conocimientos básicos, científicos y sistemáticos. Se puede contribuir a incrementar la capacidad especializada y la formación de recursos humanos orientados hacia la investigación, capaces de ofrecer soluciones a problemas fundamentales que potencialicen el desarrollo del Estado y del país, fomentando la productividad, el movimiento académico y la competitividad de los sectores estratégicos. De acuerdo a Fernando Hernández (2006), la obra se manifiesta como respuesta de los datos observados y de los que da cuenta en la propia investigación. En el caso de la investigación de la práctica de artistas profesionales, los criterios y definiciones reseñadas acotan el campo. Pero esto requiere vincularlo a planteamientos epistemológicos y metodológicos más precisos. Así mismo, se tiene en cuenta lo expresado por (Kinger, 2006), profesor de la Universidad Arizona Tucson, cuando afirma: "Las industrias cerámicas tradicionales basadas en la arcilla tienen que hacer un esfuerzo especial para beneficiarse de la nueva ciencia y de las nuevas y sofisticadas tecnologías basadas en la ciencia. El desafío consiste en aplicar la riqueza del conocimiento científico de las estructuras, procesos y principios operativos, como herramientas, para solucionar los problemas específicos a los que se enfrenta la industria cerámica". Se considera que la academia puede cooperar, en lo que le corresponde, para allanar estos problemas, más en estas pequeñas poblaciones que tienen tanta carencia de aportaciones, de procesos académicos y de acompañamiento institucional.

Fui persuadida de retomar el viejo espíritu atávico y entrar en la actividad más gratificante y formativa del ceramista: preparar la arcilla apropiada para su trabajo. $Y$, en afinidad con el maestro Jorge Fernández Chiti, ceramólogo argentino, escritor, director y fundador del Instituto de Ceramología Condorhuasi, cuando dice: "es muy importante que el alfarero sepa preparar sus propias pastas para adaptarlas a sus necesidades, 
temperaturas de cocción y gustos. Se debe disponer de, por lo menos, tres tipos de arcillas de buena calidad, puras y muy plásticas" (Fernandez, 1980). por el deseo de querer servir como multiplicadora y forjadora de los conocimientos y del posible rescate de esta tradición, en el 2011 encargamos a Charles Michel Rojas Furmanowski realizar análisis de pruebas (al diente y cal, plasticidad y trabajabilidad, rango de impurezas, alúmina, porcentajes de absorción de cocción, post-cocción y también de óxido de hierro), a tres muestras de arcilla provenientes de los yacimientos ubicados en la ladrillera de Valencia de Jesús, Pozo del Barro El Colorao y el Cerrito de las Cabras, en Patillal, con el fin de saber específicamente la acción de los diversos componentes de los barros jurisdiccionales.

Estos estudios permitieron distinguir cuáles son las arcillas aptas y cuáles no, y conjuntamente conocer que el rango máximo de temperatura es de $1.050^{\circ} \mathrm{C}$. Tienen bajísimo porcentaje de alúmina, y se soluciona adicionándole una arcilla de mayor relación alumínico y con menor proporción de impurezas, o caolín (de muy buena calidad, 20 a $26 \%$ de alúmina) para poder elevar la temperatura de cocción. Verificamos que las pastas preparadas se mantienen dentro de los rangos permitidos: estos admiten manejar una pieza en la etapa de conformación y disminuyen el riesgo de perderla a la hora de la cocción. En el 2012 elaboramos una serie de murales en pequeños formatos ${ }^{2}$, utilizando fórmulas con las arcillas de Patillal y Valencia de Jesús en proporciones de $70 \%$ + talco $25 \%$, feldespato $5 \%$, y de arena el $20 \%$, pudiendo comprobar la buena adherencia de los esmaltes a la pasta, adaptables a resultados de estándares de calidad, logrando elevar la temperatura a $1.105^{\circ} \mathrm{C}$.

Teniendo en cuenta lo que dice Ariza D. (2014): "la investigación en artes o diseño podría llegar a ser una conversación de la experiencia de quien la ejecuta y que dialoga con otros autores y experiencias en las que se expresan los caminos recorridos y por recorrer", se adelantaron pláticas con los líderes ladrilleros de tradición de la localidad de Valencia de Jesús. Estos revelaron la preocupación reinante con respecto a la crisis que atraviesa la producción ladrillera, porque no tienen materia prima con qué trabajar. En el mes de

\footnotetext{
1 Especialista en cerámica, consagrado en el estudio, desarrollo e investigación continua con materiales, esmaltes y técnicas de cocción, miembro de la Asociación de ceramistas, Artierra.

2 Esta serie fue exhibida en la sala de exposición de la Biblioteca departamental Rafael Carrillo Lúquez, en Valledupar, en el año 2012.
}

abril del 2015 visitamos este corregimiento con alumnos de la asignatura de cerámica de la Licenciatura en Arte, Folclor y Cultura de la Facultad de Bellas Artes, Universidad Popular del Cesar. Observamos un panorama desolador: enormes socavones, la arcilla existente es de tipología arenosa, y esto en nada favorece a la trabajabilidad y calidad de los productos. Solo en pequeñas islas, y al margen de la carretera que comunica a Valledupar con el norte del país, se notó que se encuentra arcilla del suelo (que es de óptima calidad y la más adecuada para el oficio) pero no está permitido extraerlas por fehacientes motivos. Con esta se trabajó en el año 2011 obteniéndose muy buenos resultados, pero en el 2015 la perspectiva es totalmente diferente. Esto es una "crónica de una muerte anunciada", para parodiar un título de nuestro nobel de literatura Gabriel García Márquez. En la conversación con los líderes ladrilleros, estos manifestaron que es una situación que han expuesto ante las autoridades competentes desde hace mucho tiempo, pero estas han hecho caso omiso. Partiendo de la base anterior, y al no existir materia prima apta para la producción, sencillamente no es posible promover el desarrollo artesanal. En consecuencia, se tomó la decisión de suspender los procesos iniciados en la población de Valencia de Jesús.

En cuanto a los interrogantes planteados, podemos contestar que en los corregimientos de Patillal y Guacoche es posible el rescate de una tradición porque cuentan con materia prima de muy buenas características y esta está acompañada de un trabajo sistematizado, con aplicaciones tecnológicas apropiadas. Luego, se puede lograr que el producto cumpla con los estándares de alta calidad exigidos por la demanda del mercado nacional e internacional.

\section{Caracterización de las arcillas}

Se estructuró una propuesta válida y científicamente fundamentada para mejorar el trabajo alfarero mediante el uso de las arcillas locales. Teniendo en cuenta que la aplicación de las pruebas y análisis realizados en el 2011 fueron favorables al mezclarlas con otros componentes arcillosos, se concluyó que se podría hacer lo propio con las de la región. Así que, en el año 2013, para complementar estos estudios, fue atendida la convocatoria interna dirigida a la comunidad académica e investigativa de la Universidad Popular del Cesar con el propósito de caracterizar las arcillas de los tres corregimientos mencionados. 


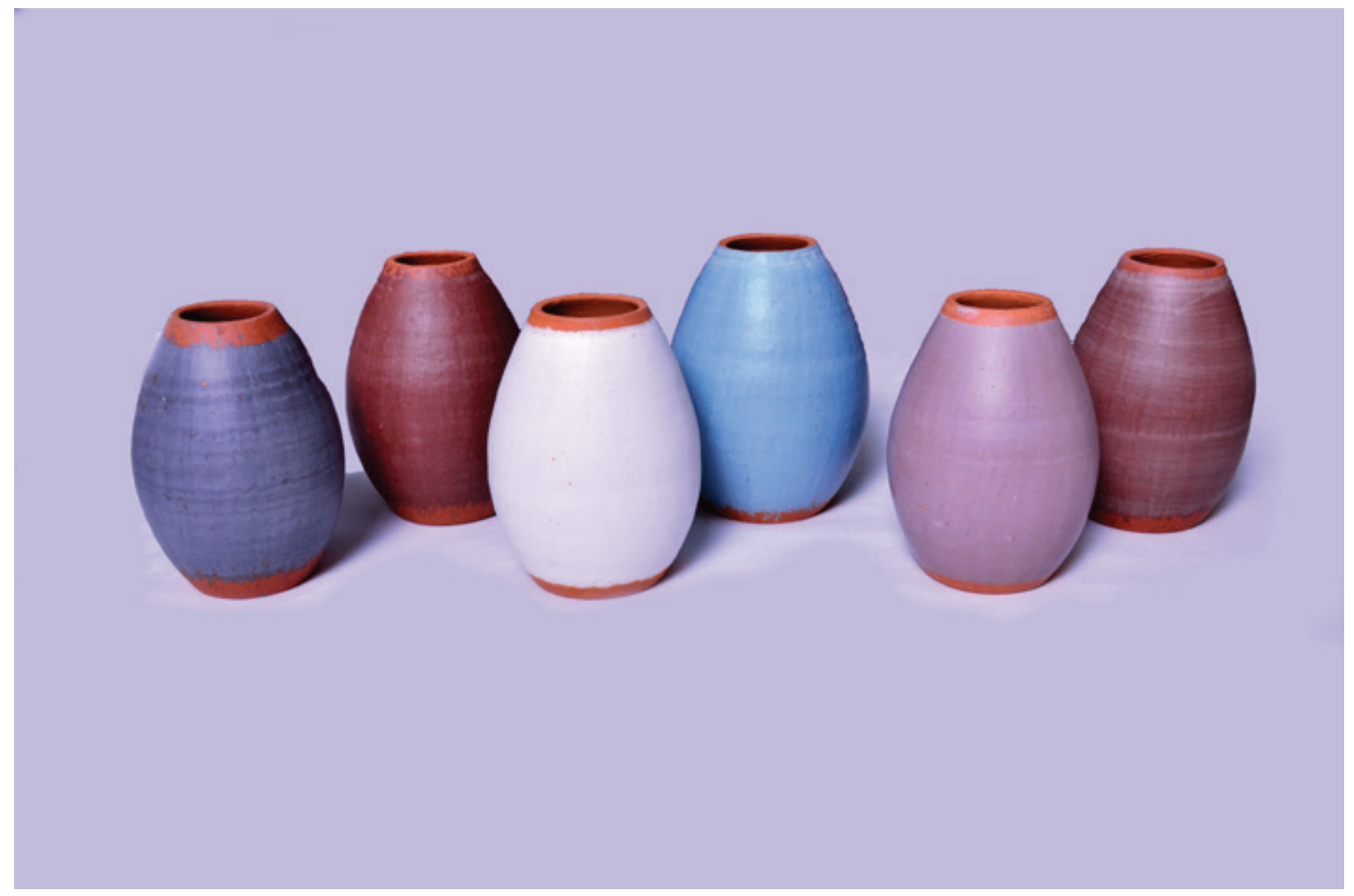

Pruebas de mezclas y engobes. Fotografía: María Cristina Cotes

Se partió de esta hipótesis: los barros usados en los corregimientos de Patillal, Valencia de Jesús y Guacoche para la confección de productos cerámicos son de muy buena calidad, unos, pero que el manejo técnico no es el indicado para obtener un producto competitivo; que otras arcillas son de tipología arenosa, pero susceptibles de mejora. Siempre asumiendo lo indicado por el maestro Fernández Chiti cuando dice que "los barros magros o arenosos se mejoran agregándoles arcilla pura o tamizándolos para eliminar el exceso de arena. (...) Casi siempre habrá que componer la pasta de acuerdo con una fórmula equilibrada, o corregir y mejorar el barro natural" (Fernández, 1978). Por consiguiente, fue necesario profundizar y complementar los estudios verificados en el 2011. Para ello se solicitó al laboratorio Magma Cerámica, en Bogotá, analizar las propiedades físicas, térmicas y de trabajabilidad. El laboratorio realizó diversas mezclas con el fin de conocer las respuestas de los barros locales; hizo pruebas para conocer la posibilidad de ser moldeado, modelado, torneado y engobado; también de preparación, aplicación y ensayo de pigmentos sobre las arcillas.

\section{Resultados}

Cuando escribo la palabra ensayo recuerdo lo expresado por Ariza, (2014) al referirse al mundo escénico: que es una experimentación, una prueba de la obra que antecede a la presentación final y que permite engranar todo el dispositivo que será entregado al espectador. Pero, en este caso, es entrar al universo de las posibilidades de la paleta de colores que se pueden obtener no solo de los pigmentos, sino de la misma arcilla. Se experimentó con la del Cerrito de la Cabras en Patillal y se elaboró una Terra Sigillata. Esta produjo un color ocre, hermoso y, de acuerdo a la intensidad de la aplicación, se consiguieron diferentes gradaciones. También se mezcló con la base utilizada para los engobes (blanco), con engobes negro, y se logró originar otras variedades de colores tierra.

Todas estas tonalidades presentaron excelentes resultados, de muy buena adherencia y textura suave. Al bruñir con un pedazo de tela de gamuza y bolsa plástica, dieron un brillo natural muy especial. En general, el 


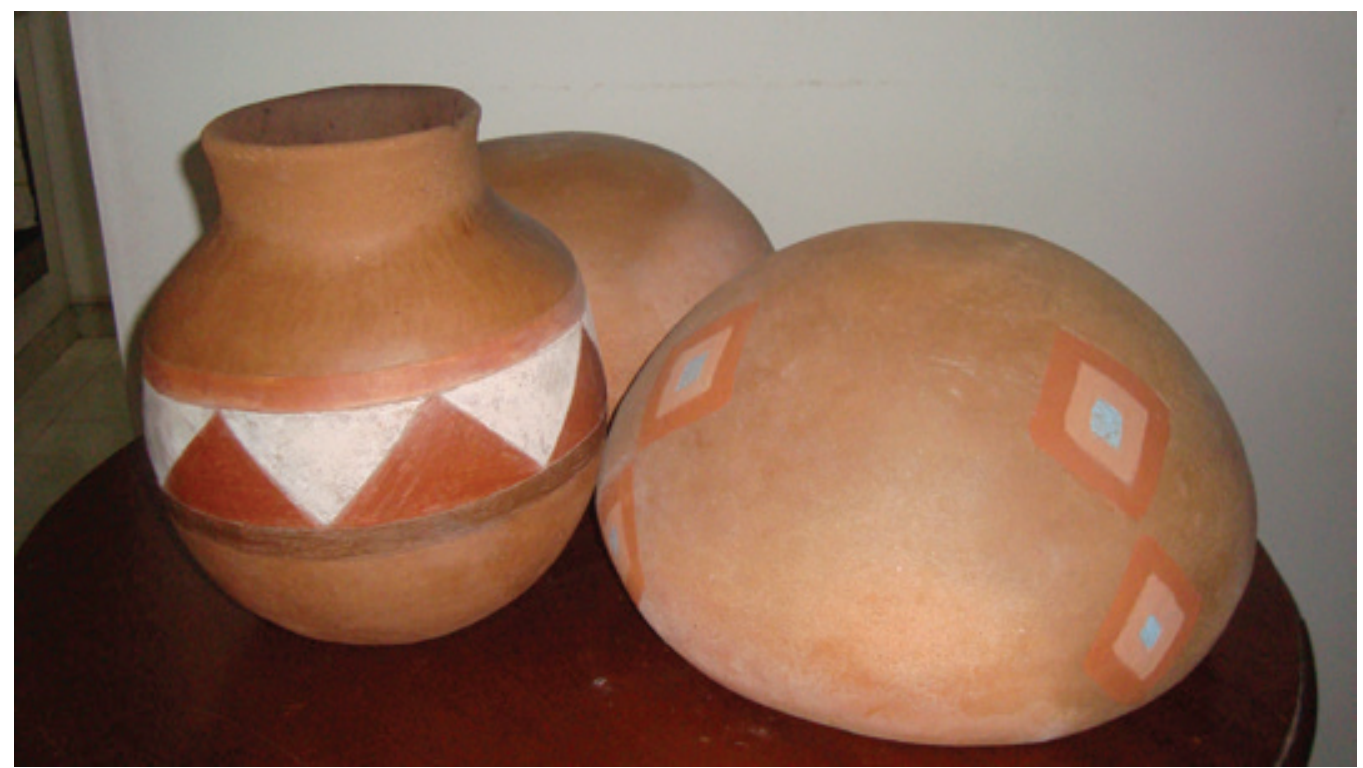

Ollas de barro después de la cocción. Fotografía: María Cristina Cotes

comportamiento de todas las arcillas fue benéfico: unas resultaron mejores que otras en el comportamiento físico-técnico y térmico, pero todas revelaron alta plasticidad. En las pruebas de las nuevas formulaciones y en las aplicaciones de engobes de colores los logros fueron muy satisfactorios, con muy buena adherencia y color homogéneo.

La excepción son las arcillas del subsuelo de Valencia de Jesús y de la ladrillera de Guacoche por su particularidad arenosa en extremo. Este exceso de arena hace que las piezas se resquebrajen y presenten fisuras, tanto en el secado, como en la cocción en diferentes temperaturas.

\section{Conclusiones}

Podemos concluir que se puede lograr un producto de buena calidad partiendo de una fórmula equilibrada en la pasta, mezclando arcillas plásticas con las moderadamente arenosas. Estas mezclas, entre sí, permiten corregir y mejorar el barro natural y lograr temperaturas desde $900^{\circ} \mathrm{C}$ hasta $1100^{\circ} \mathrm{C}$, (en horno a gas). Dado que, en las pruebas de cocción, se percibió que los hornos tradicionales de la región (cielo abierto a leña) presentan rangos de ascenso y descenso súbitos de temperatura y que escasamente llegan a los $400^{\circ} \mathrm{C}$, se construyó, de manera experimental, un "horno botella" de tiro directo, a leña, y con puerta frontal construido con ladrillos comunes en Patillal. Se pretende con esta herramienta encontrar el nivel calórico adecuado para que las piezas alcancen la dureza resistencia y durabilidad necesarias, y para ajustar el manejo de la temperatura, de los engobes y las arcillas para conseguir buenas texturas y colores, y así llegar a piezas de alta calidad y competitividad.

El Departamento Nacional de Planeación DNP (2007) contempla que para el año 2019 el sector artesanal colombiano será reconocido como un sector productivo económicamente consolidado, con alta participación en el mercado y con productos situados a nivel nacional e internacional, contribuyendo en la generación de ocupación y bienestar para la comunidad. Quisimos llevar a cabo esta investigación para llegar a ser parte de esta visión y contribuir a la solución de los problemas de una actividad artesanal en un estado de debilidad y bajo posicionamiento.

Para que estas enseñanzas no solo queden en acción dialogada aportamos un sumario formativo y orientador donde se proponen aportes complementarios que facilitan la cualificación de la alfarería regional. Orientado al adiestramiento del artesano en los distintos pasos del proceso de producción y en los métodos aplicados durante el desarrollo del proyecto de investigación, se trata de un libro didáctico impreso y en medio digital.

La cerámica es un testimonio de nuestra historia por su relación ancestral con el aire y el fuego. La reinterpretación de su inagotable potencial de transformación y, por lo tanto, de innovación, moviliza el aprendizaje interdisciplinario sobre, para y con el ser humano, y, por ser un material que se conjuga con la tierra y el agua, 
promueve un dialogo de saberes entre la academia, los artistas, los museos, los artesanos y otros espacios culturales.

\section{Referencias}

Ariza, D. (2014). Conversar, investigar, crear: la conversación como forma para evidenciar procesos de creación. Calle 14, 151 -152.

Ariza Gómez, D. (2014). Converse, research, create: conversation as a way to illustrate creative processes. Calle 14 revista de investigación en el campo del arte, 9(14), 148-156. doi:https://doi.org/10.14483/udistrital. jour.c14.2014.2.a11

Plaza, C. (2015). De lo nacional-popular a la resistencia popular: la representación y performatividad en la fiesta de los andes bolivianos. Calle 14 revista de investigación en el campo del arte, 10(15), 30-39. doi:https://doi. org/10.14483/udistrital.jour.c14.2015.1.a03

Fernández, J. (1980). Curso práctico de cerámica artística y artesanal. Buenos Aires: Condorhuasi.

(1978). El libro del ceramista. Buenos Aires, Argentina: Condorhuasi.

Hernández, F. (2006). Campos, temas y metodologías para la investigación relacionada con las artes. En M. Gómez, F.

Hernández, \& F. Pérez, Bases para un debate sobre investigación artística. Madrid: Ministerio de Educación y Ciencia.

Kinger, D. (2006). La transición de la Cerámica desde el oficio artesanal a la industria basada en la ciencia. Conferencia Cámara de Comercio de Castellón (págs. 6 - 16, 17). Castellón: Qualicer 98 Castellón.

Parra A., L. (1998). El texto absoluto en Javier Marías. LUCIA. 